essential agreement with Gest and Marrs and our current work also is in accord with this general idea. It is, however, still possible that the activation process, which was first described by Marriott et al. ${ }^{4}$, involves in some way the electron transport chain. The possibility also exists that similar mechanisms operate in vivo, but data at present available do not allow a definite conclusion to be reached.

Lister Institute of Preventative Medicine,

Chelsea Bridge Road,

London SWIW 8RH, UK

1 Davies, R. C., Gorchein, A., Neuberger, A., Sandy, J. D., and Tait, G. H., Nature, 245, is (1973).

2 Marrs, B., and Gest, H., J. Bact., 114, 1052 (1973)

${ }^{3}$ Neuberger, A., Sandy, J. D., Tait, G. H., Biochem. J., 136, 491 (1973).

4 Marriott, J., Neuberger, A., and Tait, G. H., Biochem. J., 111, 385-394 (1969).

\section{Microstructure of magnesium oxychloride cements}

SiR,--Matkovic and Young in their article on the microstructure of magnesium oxychloride cements' reported some interesting observations, and raised some points for discussion. I would like to discuss those points which have wider implications.

The authors coated their samples with a $\mathrm{Au}-\mathrm{Pd}$ layer of unstated thickness before examination in a scanning electron microscope (SEM). In the case of the metal coated specimen, SEM only reveals the outer topography of the metal layer. Figure 1 shows a section through three parallel needles each $2,000 \AA$ thick, separated by 500 $\AA$. The volume porosity of this packing is $37 \%$. Assuming that the depositing vapour had a uniform density, any layer more than $250 \AA$ (that is $\frac{1}{x}$ of the needle diameter) thick, will start to coalesce them. If the film thickness is $500 \AA$ ( $\frac{1}{4}$ of the needle diameter) the shaded areas of Fig. 1 will receive double the amount of metal necessary to fill them up. This excess metal will pile up locally, making the outer surface slightly undulated (indicated by the broken lines), and will be difficult to resolve into three. A thicker coating will make them appear monolithic. With lower porosity, a lower film thickness will be necessary to submerge them. If the needles are diverging, a metal film of proper thickness will make them appear to intergrow at the points of nearest approach, though in reality they do not. It can be seen that unless the coating thickness is adjusted to take care of particle sizc and density

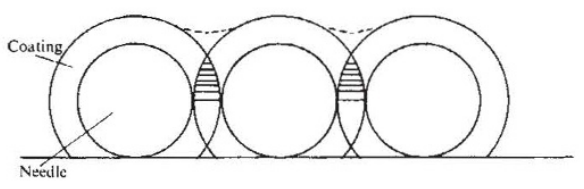

Fig. I A section through three parallel needles, each $2,000 \AA$ thick, separated from one another by $500 \AA$. The broken lines indicate the slightly undulating outer surface formed by excess metal.

of packing in the specimen, quite a misleading topography may result.

Figure $1 c$ of ref. 1 shows that the average apparent diameter of the crystals is about $1,200 \AA$. For the real diameter, twice the film thickness has to be deducted from that figure. For crystals of $1,200 \AA$ real diameter, a film thickness of $150 \AA$ or less will be required before it can be distinguished whether they are intergrowing or not. The above crystals were, however, the initial reaction products. The subsequent products are expected to be smaller and more densely packed. It is perhaps not surprising that the authors found that in older samples, the crystals were intergrown and the samp!es had a monolithic appearance. The smoothness of the cracked surfaces in their Fig. 3 can also be explained on this basis, though there are other alternatives.

The term 'intergrowth' has been used rather uncritically. There are two types of crystal-crystal intergrowth. A crystal may intergrow into another crystal without having any structural continuity, like a nail in a piece of wood. This is a special case of mechanical intergrowth. The second type of intergrowth involves structural continuity between the participating crystals. For this type of intergrowth to occur the crystals need to meet each other in definite orientations, satisfying the laws of twinning. Matkovic and Young seem to imply the second type of intergrowth. I wonder if they have tried, on this basis, an order of magnitude calculation on the probability of formation of a three dimensional load bearing structure. The result of that calculation would be quite illuminating.

Note added in proof: $\mathrm{Re}$-examination of Fig. 1c reveals isolated needles whose real diameters are equal to the stated film thickness. How many more of them were in the real sample?

\section{Yours faithfully, S. ChatTerji}

Teknologisk Institute,

Gregersensvej,

2630 Taastrup, Denmark

Matkovic. B., and Young. J. F., Nature phys. Sci., 246, 79 (1973).
Drs Young and MatKovic RePly: It is certainly true that one of the problems of SEM is the need to have a conducting layer on the surface of inorganic materials. We are aware of the inherent problems of metal coatings, but do not think it invalidates the results presented in the original article. We used comparatively light coatings $(\sim 250 \AA)$ of gold-palladium, and comparisons of such coatings with pure carbon coatings (which are transparent to secondary electrons) on samples of portland cement compounds, have not shown substantial differences in microstructural details. We therefore have confidence in the qualitative observation described in our article.

The term 'intergrowth' has been deliberately used in a vague sense and we regret any resultant misinterpretations. On the basis of SEM observations alone it is not possible to draw any definite conclusions concerning the exact interactions between crystals. We feel that, in order to explain the high mechanical strengths, these interactions must be stronger than weak Van der Waals surface forces, but may range from strong 'solid-solid contacts' postulated for hydrated portland cements $^{1}$, to true structural continuity.

Finally, we emphasise that these results are preliminary observations. We advanced our ideas in the interests of suggesting an alternative approach to the problem of strength development in cementitious systems. We realise that SEM cannot provide all the answers and we feel that, at present, attempts to make quantitative predictions may be premature.

\section{University of Illinois,}

Urbana, Illinois 61801

1 Feldman, R. F., and Sereda, P. J., Proc. fifth int. Symp. chem. Cements, Tokyo, 1958, III, 36-44 (Cement Association of Japan, Tokyo, 1969).

\section{Photosynthesis in leaves exposed to $\mathrm{SO}_{2}$ and $\mathrm{NO}_{2}$}

$\mathrm{S}^{\prime} \mathrm{R},-$-Bell and Clough ${ }^{\prime}$ have reported that the growth of $\mathrm{S} 23$ ryegrass was substantially reduced by exposure to 12 p.p.hm. (parts per hundred million) SO . Bleasdale ${ }^{2}$ grew the same variety of ryegrass in greenhouses ventilated with polluted air from out-of-doors on a suburban site in Manchester and compared the growth with that in a greenhouse ventilated with purified air. There were reductions in the dry weight of the plants amounting to $57 \%$ in the polluted atmosphere, even though the SO: concentrations were below 3 p.p. $\mathrm{hm}$. for 39 out of $42 \mathrm{~d}$ and the daily 